\title{
Quantitating Quality Measurements of Acute Pancreatitis Management
}

\author{
C. Mel Wilcox ${ }^{1} \cdot$ Paul Tarnasky $^{2}$
}

Published online: 22 July 2019

(c) Springer Science+Business Media, LLC, part of Springer Nature 2019

\section{"I know it when I see it" \\ United States Supreme Court Justice Potter Stewart, 1964}

Medicine has evolved from a solo practice model incorporating art as much as science to the practice of medicine to the current model of large networks using evidence-based decision making and algorithmic approaches to medical decision making. Being "late to the party," medicine could learn a lesson or two from industry, for which quality metrics have been used for decades. The paradigmatic "six sigma" endeavor initiated by Motorola three decades ago and later championed by Jack Welch at General Electric was aimed at process improvement by reducing variability in production methods. In so doing, defects were reduced and production times improved, ultimately enhancing the quality of its products and services. Additional benefits included increased employee morale and decreased costs, thereby increasing profits. Since then, there have been numerous programs developed to improve quality in numerous industries and manufacturing. Given the escalating expenditures for healthcare and the variable outcomes of medical care experienced among physicians and hospitals, such quality initiatives are now being demanded by payers such as Medicare. Moreover, payments to these entities will be tied to achieving specified metrics, enhancing the importance of identification and validation of these measures.

Quality can be defined and measured in many ways. For medical care, one can examine factors such as costs, mortality, morbidity, and quality of life $[1,2]$. The struggle has been to identify metrics, such that the field moves forward from subjective recognition of quality to firm data

C. Mel Wilcox

cmwilcox@uabmc.edu

1 Division of Gastroenterology and Hepatology, University of Alabama at Birmingham, 1808 7th Avenue, South, BDB 373, Birmingham, AL 35294-0007, USA

2 Methodist Dallas Medical Center, Dallas, TX, USA where these metrics measure the intended outcome, termed validity. Not surprisingly, to date most quality metrics for gastroenterologists have centered on endoscopic procedures [3], since endoscopy lends itself to objective process measures such as indications for procedure or withdrawal time at colonoscopy. The landmark study identifying adenomas at screening colonoscopy as a marker of subsequent colorectal cancer mortality has been rapidly accepted as a quality metric since the metric (adenoma detection) predicted an important outcome (colorectal cancer mortality) [4]. Unfortunately, few data are available to provide guidance regarding other metrics. For example, one can use the indication for a procedure as a quality measure, although this can easily be manipulated and likely is not truly valid. Conversely, morbidity and mortality outcomes from procedures are extremely important outcomes, although these once again can be manipulated, need to be collected prospectively, and are difficult to capture.

Until now, there has been little attention paid to assessment of quality in the area of pancreatitis management [5, 6]. It is thus with great interest that we read the study by Ketwaroo et al. published in this issue of Digestive Diseases and Sciences [7]. This study, performed by a number of recognized pancreatologists, used the well-established UCLA/Rand Delphi panel approach methodology to develop quality-of-care indicators in patients with acute pancreatitis (AP). The purpose for identifying such indicators was to understand and improve the current process of care in AP. Independently determined potential indicators were identified after review of society recommendations and a thorough literature review that formed the basis on which the authors made recommendations for inclusion or not. Most of the indicators were based upon $\mathrm{B}$ or $\mathrm{C}$ rather than $\mathrm{A}$ evidence levels. Ultimately, the group identified 22 quality indicators (QIs) that evaluated the domains of diagnosis, prevention, determination of etiology, severity, volume of fluid resuscitation, nutrition, use of antibiotics, and endoscopic or surgical management. For example, one QI asks "if a patient 
is diagnosed with AP, then liver function tests should be obtained within 24 h."

Concurrently and independently, an investigative team was assembled with a similar mission ultimately identifying 41 QIs based on literature review and which were considered valid, in the sense that the QI measures quality of care and has the potential to improve clinical practice [8]. The graded validity had to be in the "definitely" valid range in order to be ultimately considered by the group. It is fascinating that many of the QIs developed by Tarnasky and his colleagues [8] overlapped or were the same as those developed in the current study by Ketwaroo et al. [7]. Again, these ranged from appropriate diagnosis to grading of severity, antibiotic and fluid management, to the use of invasive testing such as ERCP. Importantly, both studies recognized that high-quality (grade A) data were not available for most of these metrics. Nevertheless, the strong similarities among the identified measures between the two independent groups lend further credence to their potential utility.

What's next? Can these QIs be used prospectively? As with any attempt at measurement, the metrics must be easily identifiable, simple to collect, objective, and generalizable. Some will be easy to collect by centers such as laboratory testing on admission or if patients underwent ERCP in the appropriate time frame. The initiation of nutritional supplementation and its route or the amount of intravenous fluid provided during the initial $48 \mathrm{~h}$ of admission for AP will, for example, be much more difficult to extract since record review will be required. Nevertheless, such QIs could be conveniently submitted in a standardized fashion to appropriate groups in order to document quality, particularly if necessary for the establishment of a pancreas center. Undoubtedly, much more work will be necessary to determine which of these are the most appropriate QIs, how best to collect such data and push for further high-quality prospective studies in order to confirm the correlation between indicators and outcomes such as with adenoma detection rate and mortality [4], with the hope that other more innovative
QIs will be discovered. Their development is important for a heterogeneous disease such as AP, which consumes an inordinate amount of healthcare resources worldwide and is increasing in frequency [9]. Such studies as reviewed here are a major step in moving quality assessment from appearance alone ("I know it when I see it") to an objective, standardized, and valid assessment.

\section{References}

1. Davies SM, Geppert J, McClellan M, et al. Refinement of the HCUP quality indicators. Agency for Healthcare Research and Quality (US); 2001.

2. Breyer JZ, Giacomazzi J, Kuhmmer R, et al. Hospital quality indicators: a systematic review. Int J Health Care Qual Assur. 2019;32:474-487.

3. Pohl H. Evaluating quality in endoscopy. Endoscopy. 2017;49:581-587.

4. Corley DA, Jensen CD, Marks AR, et al. Adenoma detection rate and risk of colorectal cancer and death. $N$ Engl J Med. 2014;370:1298-1306.

5. Singla A, Simons J, Li Y, et al. Admission volume determines outcome for patients with acute pancreatitis. Gastroenterology. 2009; 137:1995-01.

6. Murata A, Matsuda S, Mayumi T, et al. Effect of hospital volume on clinical outcome in patients with acute pancreatitis, based on a national administrative database. Pancreas. 2011;40:1018-1023.

7. Ketwaroo G, Sealock R, Freedman S, et al. Quality of care indicators in patients with acute pancreatitis. Dig Dis Sci. (Epub ahead of print). https://doi.org/10.1007/s10620-019-05674-8.

8. Vivian E, Cler V, Conwell D, et al. Acute pancreatitis task force on quality: development of quality indicators for acute pancreatitis management. Am J Gastroenterol. 2019. https://doi.org/10.14309 lajg.0000000000000264.

9. Peery AF, Crockett SD, Murphy CC, et al. Burden and cost of gastrointestinal, liver and pancreatic diseases in the United States: update 2018. Gastroenterology. 2019;156:254-272.

Publisher's Note Springer Nature remains neutral with regard to jurisdictional claims in published maps and institutional affiliations. 